\title{
Proliferation index revisited in neuroblastic tumors
}

\author{
Ewa lżycka-Świeszewska ${ }^{1^{\star}}$, Beata Stefania Lipska-Ziętkiewiczz ${ }^{2^{\star}}$, Elżbieta Adamkiewicz-Drożyńska ${ }^{3}$, \\ Wiesława Grajkowska ${ }^{4,5}$, Blanka Hermannn ${ }^{1}$, Ewa Bień ${ }^{3}$, Janusz Limon ${ }^{2}$ \\ *Both authors contributed equally \\ ${ }^{1}$ Department of Pathology and Neuropathology, Medical University of Gdansk, Gdansk, '2Department of Biology and Genetics, \\ Medical University of Gdansk, Gdansk, ${ }^{3}$ Department of Pediatrics, Hematology and Oncology, Medical University of Gdansk, Gdansk, \\ ${ }^{4}$ Department of Pathology, Children's Memorial Health Institute, Warsaw, ${ }^{5}$ Department of Experimental and Clinical Neuropathology, \\ Mossakowski Medical Research Centre, Polish Academy of Sciences, Warsaw, Poland
}

\begin{abstract}
Neuroblastic tumors (NB) are the most common extracranial pediatric neural crest-derived tumors, with a dismal outcome in a substantial group of patients. The study objective was to evaluate the patho-clinical correlations and prognostic impact of the proliferation index (PI) measured with two markers, Ki67 and topoisomerase II alpha (Topo2A), in a NB series. A retrospective analysis of 118 NB from 103 consecutive patients was performed. Analyzed data included tumor stage, histology, mitosis/karyorrhexis index (MKI), MYCN status, and overall survival. Patients' median follow-up period was 50 months. Ki67 and Topo2A PI were assessed immunohistochemically on representative tissue slides in hot spots. PI for Ki67 was in the range 0-72\% (median 18\%) and for Topo2A was in the range $0-58 \%$ (median 20\%), being strongly interrelated ( $r=0.83$ ). Median PIs with both markers were lower in children older than 18 months (>18 m) than in the younger patients, with $p=0.0002$ and $p=0.005$ respectively. Higher Ki67 and Topo2A correlated with metastatic stage, higher MKI, and inversely with increasing tumor differentiation. The cut-off values of PI Ki67 > 30\% and Topo2A > 20\% were associated with fatal outcome of the disease. In the subgroup of patients $>18 \mathrm{~m}$ already at cut-offs Ki67 >10\% and Topo $2 \mathrm{~A}>15 \%$ a fatal outcome was predicted by Kaplan-Meyer analysis. Cox regression analysis identified cumulative PI (joint Ki67 and Topo2A index) as an independent prognostic factor. The conclusion is that the proliferation index measured with the examined markers provides substantial prognostic information in NB, especially in infants. Pl assessment should become an element of the standard pathological checkup list of NB tumors.
\end{abstract}

Key words: neuroblastoma, proliferation index, Ki-67, topoisomerase I/ alpha, prognostic factors.

\section{Introduction}

The evaluation of the proliferation index in tumor tissue has become an important element of diagnosis and risk assessment in many types of cancer. In adult cancers, such as neuroendocrine tumors, breast carcinoma, lymphomas, prostate cancer and gliomas, the Ki-67 labeling index is included in the routine diagnostics, having therapeutic implications $[1,2,27]$. Likewise, the assessment of topoisomerase II alpha (Topo2A) expression emerges as a prolifera- 
tion marker in several cancer types, including brain tumors $[12,26,29]$. Ki-67 nuclear protein is required for maintaining cell proliferation, as it is detectable in all active phases of the cell cycle (G1, S, G2 and M) but absent in the resting phase $\mathrm{GO}[21,28]$. Accordingly, it serves for the determination of the growth fraction of a given cell population. Topo2A is one of the key enzymes responsible for maintenance and replication of genomic DNA. The expression of Topo2A increases dramatically in late $S$ phase of the cell cycle, and then decreases at the end of $M$ phase [11]. Consistent with the above, high expression of Topo2A corresponds to high proliferation activity $[5,11,12]$. The other proliferation markers, with different prognostic and predictive value, are: proliferating cell nuclear antigen (PCNA), repp86, minichromosome maintenance protein 2 (MCM2), thymidine labeling index, thymidine kinase, cyclin E, cyclin D, and cyclin inhibitors p27 and p21 [5,14,15,17,18,24].

Neuroblastoma (NB) is the most common extracranial solid tumor of early childhood, deriving from the sympatheto-adrenal lineage. It presents considerable heterogeneity in clinical behavior, ranging from spontaneous regression or maturation to metastatic spread despite aggressive therapy $[4,19,23]$. The long-term survival rates remain unsatisfactory in the high-risk group accounting for almost half of all NB cases. Several biological features and molecular abnormalities have been identified as powerful predictors of the patients' prognosis and therapeutic response. The International Neuroblastoma Risk Group (INRG) classification $[4,19]$ is based on stage of disease, patient's age, histological subtype and grade of differentiation, MYCN gene status, presence of chromosome 11q deletion and tumor ploidy. In fact, the mitosis-karyorrhexis index (MKI) remains the only marker relating to cellular proliferation in the modern diagnostic directives $[4,13]$.

Several immunohistochemical markers of proliferation in NB have been examined by a few groups $[2,3$, $5,8,15]$. It was suggested that their assessment might merit inclusion in the panel of diagnostic and prognostic criteria [6,14]. Conversely, a few reports showed no significant correlations of proliferation markers with clinico-biological features in an NB series [25]. Hence, there is still no consensus regarding significance of the proliferation index (PI) in NB. Moreover, Topo2A expression has not yet been evaluated in NB.

In NB, despite advantages in understanding of the tumor biology, there is still a need for new reliable prognostic and predictive factors. The purpose of the current study is to evaluate the utility and patho-clinical associations of the proliferation index measured with two markers.

\section{Material and methods \\ Patients}

One hundred and three NB patients aged 1-169 months (mean: 40; median: 30) at diagnosis were enrolled in the study. In six patients tumor tissue samples from consecutive surgical procedures were available. These samples were subject to all analyses except for survival probability estimation, which was limited to primary tumors. Twenty-four patients were treated with chemotherapy before biopsy. Written informed consent was obtained from the relevant guardians of the children. The International Neuroblastoma Pathology Classification (INPC) was employed for histological typing and prognostic evaluation [23]. The archival tissue slides representative for each tumor were examined by two independent observers (EIS, WG). The MKI was determined in each untreated tumor according to the Shimada criteria [23]. Clinico-pathological characteristics of the patients are shown in Table I. The mean follow-up period was 54.5 months (median: 50; range: 3-162 months). During the observation period 34 patients died of the disease.

\section{Immunohistochemistry}

The following mouse monoclonal antibodies were used for the immunohistochemical evaluation: Ki-67 (1 : 100, DAKO, F0788) and Topo2A (1 : 50, DAKO, M7186) with appropriate positive and negative controls. The standard protocol with heat-induced antigen retrieval in citrate buffer was followed. Subsequently the slides were incubated with the primary antibody for 30 minutes at room temperature. Thereafter the visualization system EnVision and DAB (DAKO) with hematoxylin staining were used. The quantitative study was carried out semi-automatically using an image analyzer system (microscope BX 51 and digital camera SP-350 with Quick Photo Camera 2.2. software, Olympus). Tumor fields with the highest number of $\mathrm{Ki}-67$ and Topo2A positive nuclei were identified (hot spots) and the total number of nuclei and immunopositive nuclei were manually indicated and counted on computer images at $200 \times$ magnification. The proportion of positive tumor cells was measured for at least 100-1000 
Table I. Clinico-pathological characteristics of patients and their corresponding 5-year overall survival rates

\begin{tabular}{|c|c|c|c|c|c|c|c|}
\hline \multirow[t]{2}{*}{ Feature } & \multirow{2}{*}{$\begin{array}{l}\text { Dichotomous } \\
\text { covariate }\end{array}$} & \multicolumn{3}{|c|}{ All patients in the study } & \multicolumn{3}{|c|}{ Patients $>18$ months of age } \\
\hline & & $n$ & 5 -year OS & $\begin{array}{c}p \text { value } \\
\text { (log-rank test) }\end{array}$ & $n$ & 5-year OS & $\begin{array}{c}p \text { value } \\
\text { (log-rank test) }\end{array}$ \\
\hline \multirow[t]{2}{*}{ Age } & $\leq 18$ months of age & 40 & $87.5 \%$ & \multirow[t]{2}{*}{0.004} & - & - & \multirow[t]{2}{*}{-} \\
\hline & $>18$ months of age & 63 & $54.1 \%$ & & - & - & \\
\hline \multirow[t]{2}{*}{ Stage } & $1,2,3,4 S$ & 64 & $93.5 \%$ & \multirow[t]{2}{*}{$<0.001$} & 31 & $90.1 \%$ & \multirow[t]{2}{*}{$<0.001$} \\
\hline & 4 & 39 & $25.2 \%$ & & 32 & $20.0 \%$ & \\
\hline \multirow[t]{2}{*}{ Prognostic group (INPC) } & $\mathrm{FH}$ & 49 & $90.0 \%$ & \multirow[t]{2}{*}{0.004} & 23 & $83.0 \%$ & \multirow[t]{2}{*}{0.026} \\
\hline & UH & 30 & $66.6 \%$ & & 19 & $57.9 \%$ & \\
\hline \multirow[t]{5}{*}{ Histological type/subtype } & GN & 9 & $87.5 \%$ & \multirow[t]{5}{*}{0.014} & 9 & $87.5 \%$ & \multirow[t]{5}{*}{0.008} \\
\hline & GNB & 20 & $61.0 \%$ & & 18 & $56.0 \%$ & \\
\hline & NB DIFF & 28 & $76.8 \%$ & & 17 & $66.5 \%$ & \\
\hline & NB POO & 40 & $68.0 \%$ & & 15 & $38.1 \%$ & \\
\hline & NB UN & 6 & $0 \%$ & & 4 & $0 \%$ & \\
\hline \multirow[t]{3}{*}{ MKI } & Low & 33 & $91.1 \%$ & \multirow[t]{3}{*}{$<0.001$} & 22 & $86.2 \%$ & \multirow[t]{3}{*}{$<0.001$} \\
\hline & Intermediate & 26 & $88.2 \%$ & & 10 & $80.0 \%$ & \\
\hline & High & 20 & $54.8 \%$ & & 10 & $30.0 \%$ & \\
\hline \multirow[t]{2}{*}{ MYCN } & Non-amplified & 79 & $81.8 \%$ & \multirow[t]{2}{*}{$<0.001$} & 43 & $71.7 \%$ & \multirow[t]{2}{*}{$<0.001$} \\
\hline & Amplified & 24 & $18.4 \%$ & & 20 & $16.2 \%$ & \\
\hline
\end{tabular}

FH - favorable histology, UH - unfavorable histology, GN - ganglioneuroma, GNB - ganglioneuroblastoma, NB - neuroblastoma, DIFF - differentiating, POO - poorly differentiated, UN - undifferentiated

cells, depending on tumor histology. Proliferation index was reported as percentage of positive cells.

\section{Statistics}

Descriptive analysis was performed for each variable, and associations between variables were analyzed using the chi-square test with adequate corrections, Mann-Whitney and Kruskal-Wallis tests when appropriate. The effect of on long-term survival rates were analyzed using Kaplan-Meier survival probability estimates and log-rank tests. The parameters shown by univariate analysis to be significant were also compared by means of multivariate Cox regression analysis for multiple proportional hazards using a stepwise (backward and forward) conditional approach. All calculations were performed using Statistica 9.0 (StatSoft Inc.) software.

\section{Results}

\section{Characteristics of the analyzed group}

Age at diagnosis over 18 months (>18 m), metastatic disease (INSS stage 4), unfavorable histology subtype and MYCN amplification were found to be of prognostic significance (Table I). The distribution of patients with respect to these parameters has proven good representation of all the subtypes of the disease in the analyzed cohort.

\section{Correlation of PIs with clinical, pathological and molecular parameters}

Ki-67 and Topo2A-positive nuclei were distributed unevenly within the tumors, forming areas with higher concentrations ("hot spots") (Figs. 1A-B). In the whole series of tumors the mean value of Ki-67 was $22.5 \%$ (median: 18 ; range: $0-72 \%$ ); and $20.7 \%$ for Topo2A (median: 20; range: $0-58 \%$ ). These two markers were strongly interrelated (Spearman's correlation coefficient $r=0.83, p<0.05$; Fig. 2 ). In the NB Schwannian stroma poor subgroup the mean Ki-67 value was $29.1 \%$ (median: 27 ; range: $4-72$ ) and $25.6 \%$ for Topo2A (median: 25; range: $4-58 \%$ ), being significantly higher than in Schwannian stroma rich tumors ( $p<0.0001) .6 / 113$ tumors were negative for $\mathrm{Ki}-67$ and 4/113 were negative for Topo2A, including three cases negative for both markers. 

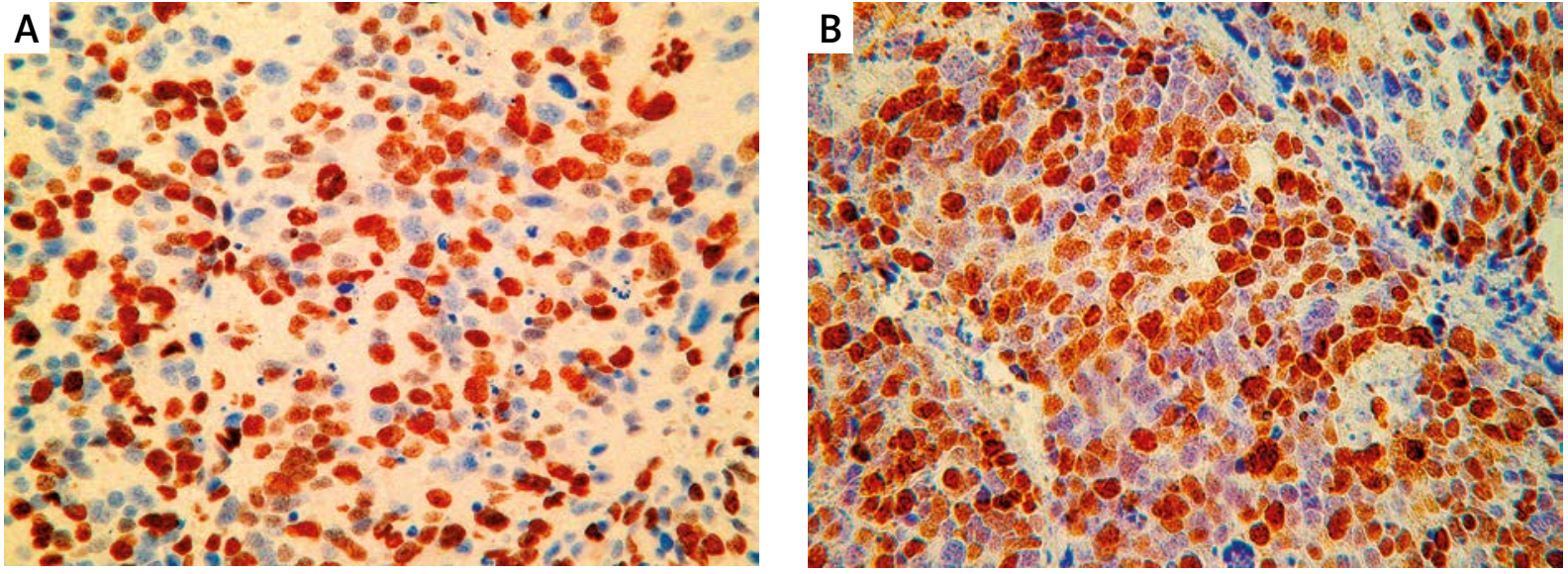

Fig. 1. Ki-67 (A) and Topo2A (B) labeling in Schwannian stroma-poor neuroblastoma in hot spot area (Ki-67, Topo2A immunostaining, 200x).

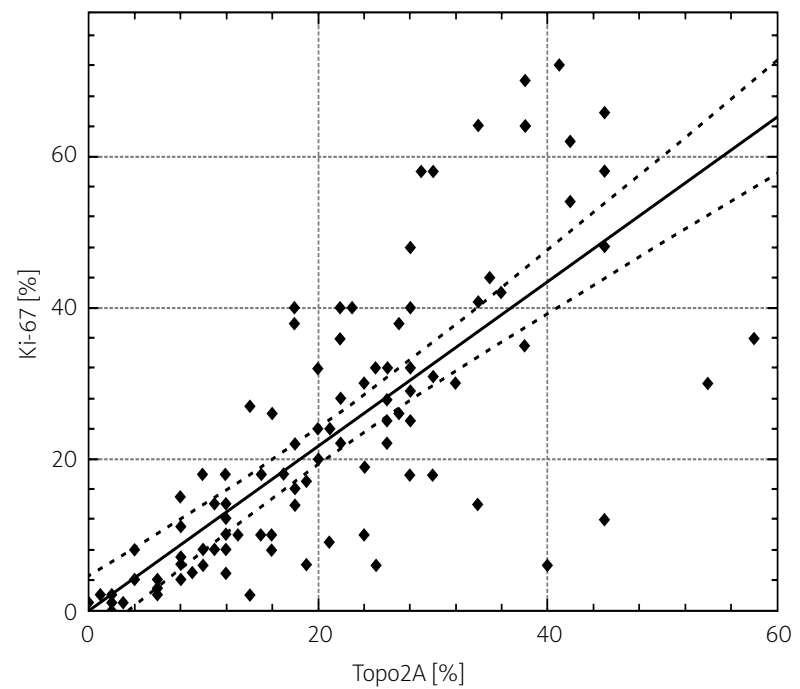

Fig. 2. Scatterplot diagram illustrating distribution of Ki-67 and Topo2A labeling indices in the whole series of NB tumors $(r=0.83 ; p<0.05)$.

Both PIs were found significantly lower in the group of patients $>18 \mathrm{~m}$ (median: $27.5 \%$ vs. $10 \%, p<0.001$ for Ki-67 and median $23.5 \%$ vs. $14 \%, p=0.005$ for Topo2A). In view of this, we further analyzed PIs with respect to the age group.

In the whole series, higher Ki-67 and Topo2A correlated with higher INSS stage. PIs were low in stages 2 and 3, intermediate in stages 1 and 4s, and high in stage 4. Patients with metastatic disease had significantly higher PIs ( $p=0.008, p=0.01$ for Ki-67 and Topo2A, respectively). PIs were inversely correlated with increasing tumor differentiation. The highest PI disclosed undifferentiated and poorly differentiated
NB, while the lowest or even null index concerned ganglioneuroma. Analogously, cases with unfavorable histology according to INPC were found to have significantly higher PIs ( $p<0.001$ for both markers). PIs strongly correlated with MKI. There was no difference in mean PI values between the non-treated and pretreated cases. Conversely, the samples from consecutive operations of a single patient were characterized by a considerable drop in MKI, Ki67 and Topo2A values. Adrenal localization of the primary tumors was characterized by significantly higher PIs. MYCN amplification was found to be associated with higher PIs, but only for Topo2A did the observation reach the level of statistical significance $(p=0.025)$.

\section{Survival analyses}

PIs were predictive of poor prognosis at the cut-off values of $30 \%$ for $\mathrm{Ki}-67$ and $20 \%$ for Topo $2 \mathrm{~A}$ (Table II, Fig. 3). The correlation remained significant in the subgroup of 79 chemonaive tumors. 27 patients (26\%) had both indices - referred to subsequently as cumulative PI (cumPI) - exceeding the defined cut-off values, and these had the worst prognosis (Fig. 3C). Furthermore, cumPI had prognostic significance in the MYCN non-amplified group (5-OS rates $85.4 \%$ vs. $68.2 \%, p=0.048)$. In the unfavorable histology (UH) group, the cumPI was related to survival (5-OS rates $82.4 \%$ vs. $45.6 \%, p=0.01$ ). In the stroma poor group the cumPI value was also highly predictive of survival (5-OS rates $79.2 \%$ vs. $45.2 \%$; $p=0.004$ ). In stage 4 , a borderline tendency for worse survival in the group with intense proliferation established cumPI $(p=0.06)$. 
Table II. Proliferation markers in the analyzed series of NB tumors and their corresponding 5-year overall survival rates

\begin{tabular}{|c|c|c|c|c|c|c|c|}
\hline \multirow[t]{2}{*}{ Feature } & \multirow{2}{*}{$\begin{array}{l}\text { Dichotomous } \\
\text { covariate }\end{array}$} & \multicolumn{3}{|c|}{ All patients in the study } & \multicolumn{3}{|c|}{ Patients $>18$ months of age } \\
\hline & & $n$ & 5-year OS & $\begin{array}{c}p \text { value } \\
\text { (log-rank test) }\end{array}$ & $n$ & 5-year OS & $\begin{array}{c}p \text { value } \\
\text { (log-rank test) }\end{array}$ \\
\hline \multirow[t]{2}{*}{ Ki-67 } & $\leq 30 \%$ & 73 & $73.6 \%$ & 0.01 & 49 & $64.4 \%$ & 0.001 \\
\hline & $>30 \%$ & 30 & $50.1 \%$ & & 14 & $20.4 \%$ & \\
\hline \multirow[t]{2}{*}{ Topo2A } & $\leq 20 \%$ & 54 & $74.1 \%$ & 0.047 & 40 & $67.4 \%$ & 0.003 \\
\hline & $>20 \%$ & 49 & $59.1 \%$ & & 23 & $31.9 \%$ & \\
\hline \multirow[t]{2}{*}{ Ki-67 and Topo2A } & other & 76 & $74.5 \%$ & 0.002 & 50 & $65.3 \%$ & $<0.001$ \\
\hline & $>30 \%$ and $>20 \%$ & 27 & $45.2 \%$ & & 13 & $13.8 \%$ & \\
\hline
\end{tabular}

\section{Multivariate analyses}

In the multivariate Cox regression analysis the initial testing was performed using two models. The first, comprising the whole series of tumors, used six covariates identified in univariate analyses (age < 18 months, stage 4, histology subtype, MYCN status, Ki-67 > 30\% and Topo2A > 20\%). Here, only stage 4 and age were identified as independent predictors of OS. In the second model, only the pre-treated cases were analyzed. Accordingly, two additional covariates (histological subtype and MKI) were added, and again, only stage 4 and age were significant. In the second step, individual PIs were replaced by the cumPI, yet still the analysis retrieved the same predictors. Finally, the stepwise conditional approach was used to identify the minimal set of markers predictive for OS. After backward elimination of the least significant covariates, the minimal models were built. For the whole series the independent prognostic factors were age, stage 4 and the cumPI (Table IIIA), while for the series of non-treated tumors the minimal model comprised age and INSS stage 4 only. Inclusion of information on MYCN status did not improve the model, probably due to a strong association between stage 4 and MYCN amplification (56\% of patients with stage 4 harbored MYCN amplification; $p<0.001)$.

\section{Significance of Pls in the group of children diagnosed over 18 months of age}

The above correlations between PIs and stage 4, histological subtype, MKI and MYCN amplification were all also significant in the subgroup of $>18 \mathrm{~m}$ patients, with the statistical power higher than in the whole series. Conversely, no correlations were detected in the youngest children. In the patients $>18 \mathrm{~m}$ the cut-off values as identified for the whole series were also predictive for long-term survival (Table II). However, according to the significantly lower mean values of Pls in this group, the cut-off values lowered to $10 \%$ for $\mathrm{Ki}-67$ and $15 \%$ for Topo $2 \mathrm{~A}$ achieved even higher statistical significance (Figs. 3D-E). The correlations remained significant in the non-treated subgroup and in Schwannian stroma poor patients. Twenty-six patients (41\%) had both indices exceeding the cut-off values, with the worst 5 -year overall survival (5-OS) (Fig. 3F). The multivariate Cox regression analyses were performed using the models as above. It identified INSS stage 4 and Ki-67 $>10 \%$ as the independent predictors of OS in the $>18 \mathrm{~m}$ group. The second, performed on non-treated $>18 \mathrm{~m}$ cases, identified INSS stage 4 as the only independent predictor. In the second step, individual PIS were replaced by the cumPI. The cumPI appeared as an independent prognostic factor in the whole series of $>18 \mathrm{~m}$ tumors but not in the pre-treatment subgroup. Eventually, the stepwise conditional approach through backward elimination of the least significant covariates allowed the minimal set of markers predictive for OS to be established. For the whole series of $>18 \mathrm{~m}$ tumors the independent prognostic factors were INSS stage 4 and the cumPI (Table IIIB), while for the series of non-treated $>18 \mathrm{~m}$ tumors the minimal model comprised INSS stage 4 only.

\section{Discussion}

The basic, direct method of cellular proliferation assessment in tumor tissue samples is the mitotic 
A

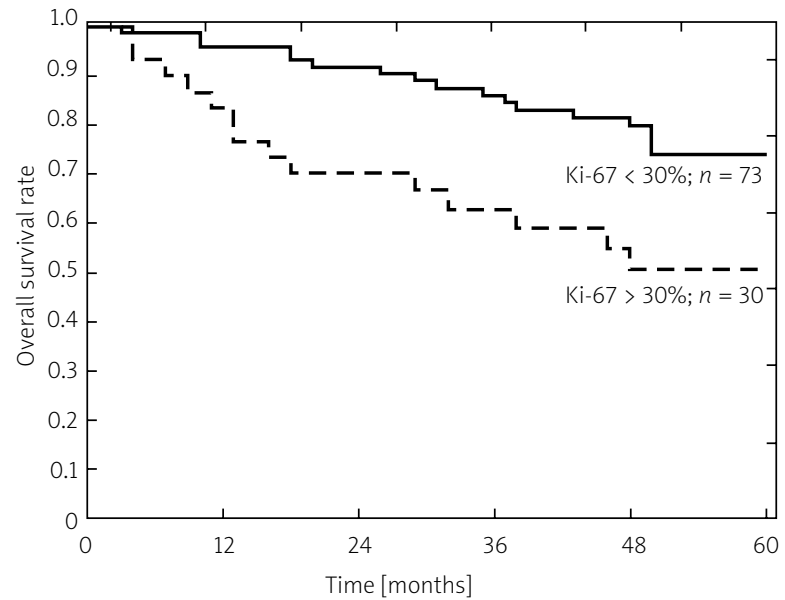

B

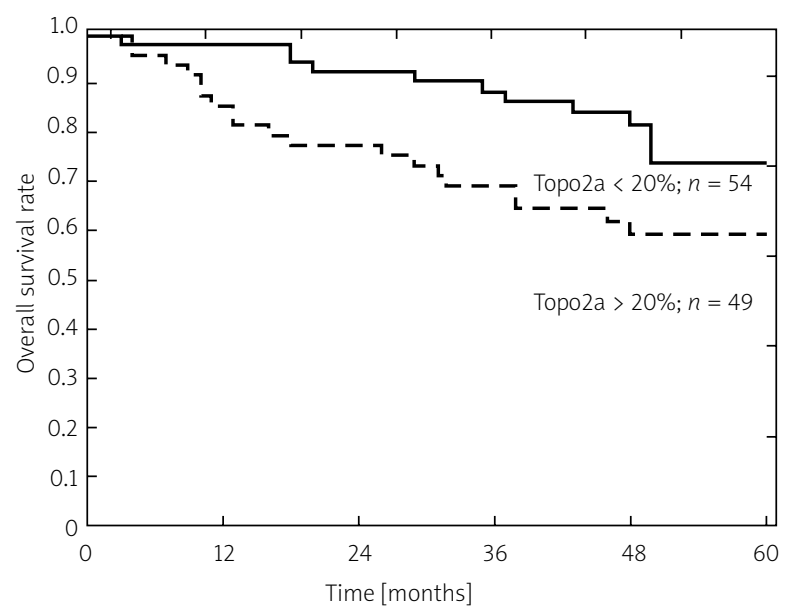

C

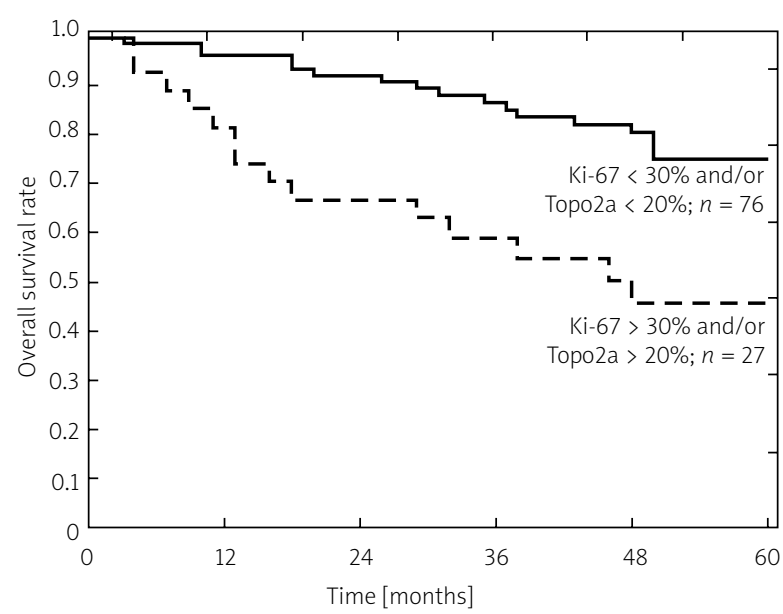

D

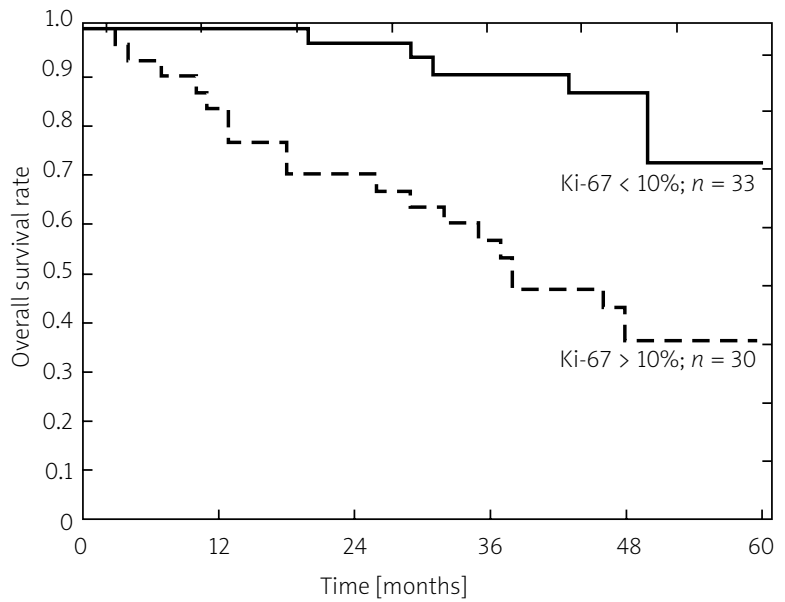

$\mathrm{E}$

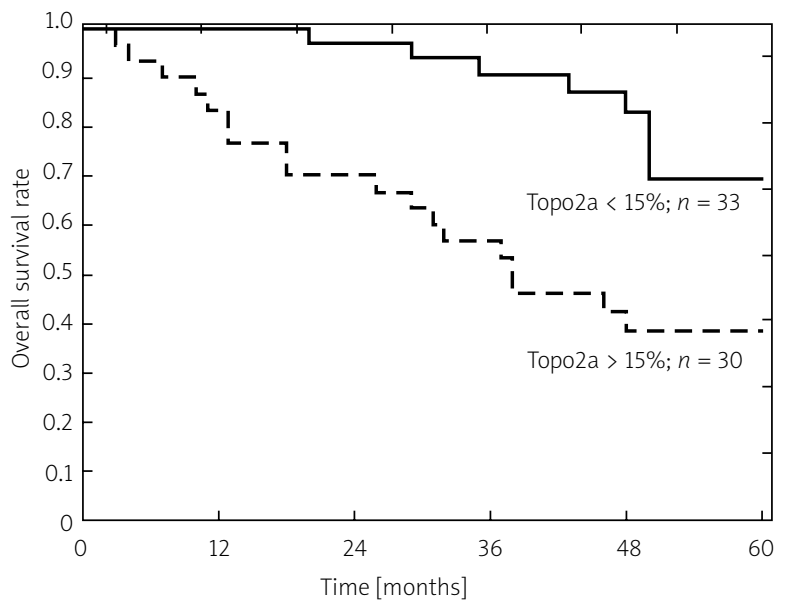

$\mathbf{F}$

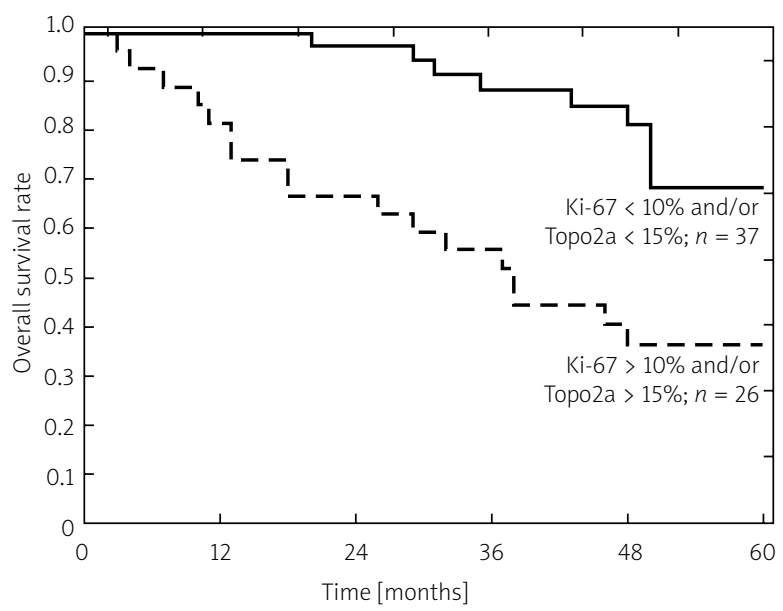

Fig. 3. Kaplan-Meier estimates of 5-year OS for neuroblastic tumors (NB) patients (A, B, C) and 5-year OS for NB patients over 18 months of age (D, E, F) with respect to PI measured with Ki-67 (A, D), Topo2A (B, E) and the cumulative PI (C, F). 
Table III. Independent prognostic factors of the long-term OS rates identified by multivariate Cox hazard regression model $A$ in the whole series of tumors and $B$ in the group of patients diagnosed over the age A of 18 months (overall significance of the models at $p<0.001$ )

\begin{tabular}{|lccc|}
\hline Feature & HR & $95 \% \mathrm{Cl}$ & $\begin{array}{c}p \text { value } \\
\text { (Wald statistics) }\end{array}$ \\
\hline Age $<18$ months & 0.1 & $0.04-0.4$ & $<0.001$ \\
\hline INSS stage 4 & 3.5 & $1.7-7.1$ & $<0.001$ \\
\hline Ki-67 $>$ 30\% and Topo2A $>$ 20\%; cumulative PI & 2.1 & $1.02-4.4$ & 0.045 \\
\hline
\end{tabular}

\section{B}

\begin{tabular}{|lccc|}
\hline Feature & HR & $95 \% \mathrm{Cl}$ & $\begin{array}{c}p \text { value } \\
\text { (Wald statistics) }\end{array}$ \\
\hline INSS stage 4 & 7.2 & $2.6-20.0$ & $<0.001$ \\
\hline Ki-67 > 10\% and Topo2A > 15\%; cumulative PI & 2.4 & $1.1-5.2$ & 0.03 \\
\hline
\end{tabular}

HR - hazard ratio, Cl - confidence interval

count or mitotic index. The mitotic count is used in many tumors as a diagnostic (smooth muscle tumors, meningiomas) or prognostic factor useful in the therapeutic approach (GISTs, neuroendocrine tumors, breast cancer) $[16,18,27]$. In pathological reports of many tumors, parallel data on the mitotic count and proliferative index are needed nowadays $[1,7,18]$. The mitotic count represents only a small fraction of cells at a cell cycle point, while markers of proliferation detectable by immunohistochemistry can identify the entire active cell population. Among several markers of proliferation, Ki67 is the most popular and reliable one. Topoisomerase 2A was accepted as a proliferative marker in gliomas and a few other cancers $[17,29]$. PI may be evaluated in the highest staining region (hot spot $\mathrm{PI}$ ), or as an average count from randomly chosen fields (random $\mathrm{PI}$ ). Most often Ki67 is evaluated based on the hot spot approach, which is a more reliable procedure $[14,15,25,27]$. Depending on the type of the tumor, large discrepancies between the number of cells per field and hence random selection of fields for PI evaluation might bias its score and produce unrepeatable results. Several tumor-type criteria for PI establishment have been elaborated. Digital image analysis systems can further improve the reliability of $\mathrm{PI}$ assessment $[1,13,26]$.

Neuroblastic tumors constitute a heterogeneous group of tumors, presenting up-regulated proliferation, prolonged cell survival, inhibited apoptosis and disturbed neuronal differentiation [19]. The sig- nificance of the proliferation index in NB has been elaborated in several studies, but the MKI is the only parameter related to proliferation to be generally accepted in the last 30 years $[4,19,23]$. The Shimada classification originally published in 1984 , with a few subsequent modifications, is a widely accepted, histopathological age-based prognostic system for NB $[10,23]$. The MKI was introduced in this system, as the density sum of mitotic and karyorrhectic cells in 5000 neoplastic cells. The MKI was evaluated and critically discussed soon after it was introduced into practice [10]. The biological nature of karyorrhectic cells is debatable, but a high MKI in stroma-poor tumors is an indicator of poor prognosis $[9,10]$. The karyorrhectic cells were shown to be either proliferating or undergoing apoptosis [9,25]. Furthermore, a high cellular density of proliferating cells correlates with poor prognosis, whereas a high density of apoptosis indicates a favorable outcome [13]. Moreover, the assessment of mitotic rate in an NB tumor section is time-consuming and can be difficult to perform due to the specificity of tumor histology. In undifferentiated and poorly differentiated NB, application of the mitotic count is still controversial because of the high cellularity and mutual resemblance of mitotic and karyorrhectic cells $[9,10,25]$.

In the current study, both of the analyzed PIs strongly correlated with the MKI (Fig. 1). Previously, a Spanish group also reported a significant association between the MKI and Ki-67 index [3]. Conversely, in their simplified dichotomous (low vs intermedi- 
ate and high MKI) analysis performed on a smaller group of 60 NB tumors, Uccini et al. [25] observed such a correlation only in the subgroup of MYCN amplified tumors. Moreover, contrary to MKI, immunohistochemical markers can be assessed not only in the pre-treatment specimens but also in tumors that were resected after inductive chemotherapy [8]. No difference in mean PI values was found between pre-treated and non-treated cases - which is in line with a previous report by Krams et al. [14,15]. In our study, we also analyzed PIs in tissue samples from consecutive biopsies of single patients. Here we observed a considerable drop in PI values after chemotherapy parallel to tumor maturation. The two surviving patients were the only ones with non-metastatic disease. Recently, it has been proposed that examination of NB samples after the inductive chemotherapy gives prognostic information [8]. In general, a shift from an intermediate/high to low MKI was observed. Following induction therapy, persistence of intermediate/high tumor MKI and high proliferative activity portends a poor outcome [8].

Rudolph et al. [22] proposed Ki-67 as a reliable means for the assessment of the tumor growth fraction in NB. Ki-67 was significantly correlated with histopathological grade and predictive for long-term OS at a cut-off level of $25 \%$. Also, the Ki-67 index was significantly higher in stage 4 cases, and the $\mathrm{Ki}-67 \mathrm{PI}$ enabled risk stratification in tumors with and without MYCN amplification. Moreover, the $30 \%$ cut-off value was identified in Cox regression analysis as an independent prognostic factor along with stage $[14,15,22]$. Del Carmen et al. confirmed a significant correlation with histologic subtype, MKI and disease stage [6]. Later, analysis of a 182 Spanish NB registry revealed that the death rate increased markedly with the index exceeding 30\% [3]. Our results are in line with the abovementioned reports.

Our study is the first to analyze Topo2A expression in NB. It has been suggested that due to selective expression during the cell cycle Topo2A might provide a better estimate of the number of actively cycling cells than Ki-67 [11]. The assessment of whether the prognostic impact of Topo2A is due to its value as a predictor of response to a treatment-based regimen or as a pure prognostic factor is still difficult to establish $[5,12,29]$. Similar to the studies on other cancers $[1,5,18,21]$, we have shown that NB patients with high Topo2A PI experience significantly shorter OS. Also, Topo2A strongly correlat- ed with Ki-67 (Fig. 1) and MKI. The addition of this parameter to the Ki67 proliferation index allowed for more precise prediction of patient risk in multivariate analyses (cumulative PI). Interestingly, Korija et al. [13] found that the anti-Ki-67 assessed proliferation activity was significantly higher in MYCNamplified than in non-amplified hotspots. The proliferation indices of the hotspots also had a significant correlation with the prognosis and histological type. Hotspot focusing provides a means of analyzing proliferation-associated markers in neuroblastomas, and together with the FISH detection of the MYCN copy number enables an easy and reliable examination of MYCN status in neuroblastomas.

The novelty and the impact of our study is the consequence of two particularities. Firstly, the analysis of the percentages of Ki-67-positive and/or Topo2A-positive cells as a continuous value allowed us to avoid a potential bias inherent in the use of an arbitrary cutoff point, and supported the association of high $\mathrm{Ki}-67$ and or Topo2A expression with a high risk of death due to disease. From the practical point of view, values above $30 \%$ for $\mathrm{Ki} 67$ and 20\% for Topo2A were related to a dismal outcome in the whole group and also in the stroma poor NB subgroup.

Furthermore, for the first time the analysis of PIs with respect to the age of the patients as a stratifying parameter was performed. The age of NB patients is an important prognostic and risk factor with the cutoff eventually defined recently at 18 months of age $[4,20]$. Age can be thought of as a surrogate risk factor for genetic or biologic markers of risk that are yet undiscovered or unproven $[19,20]$. Of note, del Carmen Mejia et al. [6] also observed the peculiar correlation between age at diagnosis and Ki-67 expression, but the authors could not propose a consistent explanation for this. In the younger subgroup of our patients, moderate proliferation is not related to adverse prognosis. Probably it is just a manifestation of the ongoing processes of maturation of neoplastic cells $[10,23]$. Conversely, the older patients have, on average, lower proliferation. However, a fraction of them will have poorly differentiated histology and intense proliferation with unfavorable outcome of the disease. This double-faced nature of proliferation in NB converts into two cut-off values of PIs, age-dependent, identified in the current study.

The presented analysis of PIs provides further insight into the distinctive biological heterogeneity of NB, especially with respect to the age of the 
patient. It seems that the immunohistochemically assessed PI should be introduced in parallel to the MKI to the routine diagnostics of NB tumors, especially the Schwannian stroma poor group. However, the significance of $\mathrm{Pl}$ needs further validation on larger prospective groups of patients.

\section{Acknowledgments}

The study was supported by the Polish Ministry of Science and Higher Education grant N401 197339.

\section{Disclosure}

\section{Authors report no conflict of interest.}

\section{References}

1. Arnold CN, Nagasaka T, Goel A, Scharf I, Grabowski P, Sosnowski A, Schmitt-Gräff A, Boland CR, Arnold R, Blum HE. Molecular characteristics and predictors of survival in patients with malignant neuroendocrine tumors. Int J Cancer 2008; 123: 1556-1564.

2. Berney DM, Gopalan A, Kudahetti S, Fisher G, Ambroisine L, Foster CS, Reuter V, Eastham J, Moller H, Kattan MW, Gerald W, Cooper C, Scardino P, Cuzick J. Ki-67 and outcome in clinically localised prostate cancer: analysis of conservatively treated prostate cancer patients from the Trans-Atlantic Prostate Group study. Br J Cancer 2009; 100: 888-893.

3. Burgues O, Navarro S, Noguera R, Pellín A, Ruiz A, Castel V, Llom bart-Bosch A. Prognostic value of the International Neuroblastoma Pathology Classification in neuroblastoma (Schwannian stroma-poor) and comparison with other prognostic factors: a study of 182 cases from the Spanish neuroblastoma registry. Virchows Arch 2006; 449: 410-420.

4. Cohn SL, Pearson AD, London WB, Monclair T, Ambros PF, Brodeur GM, Faldum A, Hero B, lehara T, Machin D, Mosseri V, Simon T, Garaventa A, Castel V, Matthay KK. The International Neuroblastoma Risk Group (INRG) Classification System: An INRG Task Force Report. J Clin Oncol 2009; 27: 289-297.

5. Colozza M, Azambuja E, Cardoso F, Sotiriou C, Larsimont D, Piccart MJ. Proliferative markers as prognostic and predictive tools in early breast cancer: where are we now? Ann Oncol 2005; 16: 1723-1739.

6. Del Carmen Mejía M, Navarro S, Pellín A, Ruíz A, Castel V, Llom bart-Bosch A. Study of proliferation and apoptosis in neuroblastoma. Their relation with other prognostic factors. Arch Med Res 2002; 33: 466-472.

7. Gauchotte G, Vigouroux C, Rech F, Battaglia-Hsu SF, Soudant M, Pinelli C, Civit T, Taillandier L, Vignaud JM, Bressenot A. Expres sion of minichromosome maintenance MCM 6 protein in meningiomas is strongly correlated with histologic grade and clinical outcome. Am J Surg Pathol 2012; 36: 283-291.

8. George RE, Perez-Atayde AR, Yao X, London WB, Shamberger RC Neuberg D, Diller L. Tumor histology during induction therapy in patients with high-risk neuroblastoma. J Clin Oncol 2011; 29: 4358-4364.
9. Gestblom C, Hoehner J, Pahlman S. Proliferation and apoptosis in NB: subdividing the mitosis karryorhexis index. Eur J Cancer 1995; 31A: 458-463.

10. Joshi VV1, Rao PV, Cantor AB, Altshuler G, Shuster JJ, Castleberry RP. Modified histologic grading of neuroblastomas by replacement of mitotic rate with mitosis karyorrhexis index. A clinicopathologic study of 223 cases from the Pediatric Oncology Group. Cancer 1996; 77: 1582-1588.

11. Kellner U, Sehested M, Jensen PB, Gieseler F, Rudolph P. Culprit and victim - DNA topoisomerase II. Lancet Oncol 2002; 4: 235-243.

12. Kim EJ, Lee YS, Kim YJ, Kim MJ, Ha YS, Jeong P, Lee OJ, Kim WJ. Clinical implications and prognostic values of topoisomerase-II alpha expression in primary non-muscle-invasive bladder cancer. Urology 2010; 75: 1516.e9-e13.

13. Korja M, Finne J, Salmi TT, Kalimo H, Karikoski R, Tanner M, Isola J, Haapasalo H. Chromogenic in situ hybridization-detected hotspot MYCN amplification associates with Ki-67 expression and inversely with nestin expression in neuroblastomas. Mod Pathol 2005; 18: 1599-1605.

14. Krams M, Heidebrecht HJ, Hero B, Berthold F, Harms D, Parwaresch R, Rudolph P. Repp86 expression and outcome in patients with Neuroblastoma. J Clin Oncol 2003; 21: 1810-1818.

15. Krams M, Hero B, Berthold F, Parwaresch R, Harms D, Rudolph P. Proliferation marker Ki-S5 discriminates between favorable and adverse prognosis in advanced stages of Neuroblastoma with and without MYCN amplification. Cancer 2002; 94: 854-861.

16. Larysz D, Blamek S, Rudnik A. Clinical aspects of molecular biology of pituitary adenomas. Folia Neuropathol 2012; 50: 110-117.

17. Lind-Landström T, Varughese RK, Sundstrøm S, Torp SH. Expression and clinical significance of the proliferation marker minichromosome maintenance protein $2(\mathrm{Mcm} 2)$ in diffuse astrocytomas WHO grade II. Diagn Pathol 2013; 8: 67.

18. Lowe K, Khithani A, Liu E, Winston T, Christian D, Saad J, Jeyarajah DR. Ki-67 labeling: a more sensitive indicator of malignant phenotype than mitotic count or tumor size? J Surg Oncol 2012; 106: 724-727.

19. Maris JM. Recent advantages in neuroblastoma. N Engl J Med 2010; 362: 2202-2211.

20. Moroz V, Machin D, Faldum A, Hero B, lehara T, Mosseri V, Ladenstein R, De Bernardi B, Rubie H, Berthold F, Matthay KK, Monclair T, Ambros PF, Pearson AD, Cohn SL, London WB. Changes over three decades in outcome and the prognostic influence of age-at-diagnosis in young patients with neuroblastoma: A report from the International Neuroblastoma Risk Group Project. Eur J Cancer 2011; 47: 561-571.

21. Preusser M, Hoeftberger R, Woehrer A, Gelpi E, Kouwenhoven M, Kros JM, Sanson M, Idbaih A, Brandes AA, Heinzl H, Gorlia T, Hainfellner JA, van den Bent M. Prognostic value of Ki67 index in anaplastic oligodendroglial tumours - a translational study of the European Organization for Research and Treatment of Cancer Brain Tumor Group. Histopathology 2012; 60: 885-894.

22. Rudolph P, Lappe T, Hero B, Berthold F, Parwaresch R, Harms D, Schmidt D. Prognostic significance of the proliferative activity in neuroblastoma. Am J Path 1997; 150: 133-145.

23. Shimada H, Ambros IM, Dehner LP, Hata J, Joshi VV, Roald B, Stram DO, Gerbing RB, Lukens JN, Matthay KK, Castleberry RP. 
Ewa Iżycka-Świeszewska, Beata Stefania Lipska-Ziętkiewicz, Elżbieta Adamkiewicz-Drożyńska, Wiesława Grajkowska, Blanka Hermann, Ewa Bień, Janusz Limon

The International Neuroblastoma Pathology Classification

(The Shimada system). Cancer 1999; 86: 364-372.

24. Taran K, Owecka A, Kobos J. Prognostic importance of cyclin-E1 expression in neuroblastoma tumors in children. Pol J Pathol 2013; 64: 149-52

25. Uccini S, Colarossi C, Scarpino S, Boldrini R, Natali PG, Nicotra MR, Perla FM, Mannarino O, Altavista P, Boglino C, Cappelli CA, Cozzi D, Donfrancesco A, Kokai G, Losty PD, McDowell HP, Dominici C. Morphological and molecular assessment of apoptotic mechanisms in peripherial neuroblastic tumours. Br J Cancer 2006; 95: 49-55.

26. Yang QC, Zhu Y, Liou HB, Zhang XJ, Shen Y, Ji XH. A cocktail of MCM2 and TOP2A, p16INK4a and Ki-67 as biomarkers for the improved diagnosis of cervical intraepithelial lesion. Po J Pathol 2013; 64: 21-27.

27. Yerushalmi R, Woods R, Ravdin PM, Hayes MM, Gelmon KA. Ki67 in breast cancer: prognostic and predictive potential. Lancet Oncol 2010; 11: 174-183.

28. Zawrocki A, Iżycka-Świeszewska E, Papierz W, Liberski PP, Zakrzewski K, Biernat W. Analysis of the prognostic significance of selected morphological and immunohistochemical markers in ependymomas, with literature review. Folia Neuropathol 2011; 49: 94-102.

29. Zhao H, Yu H, Liu Y, Wang Y, Cai W. DNA topoisomerase II-alpha as a proliferation marker in human gliomas: correlation with PCNA expression and patient survival. Clin Neuropathol 2008; 27: 83-90. 